\title{
Ranking de instituciones más productivas del sector sanitario español
}

\author{
Por Grupo Scimago
}

LA PRODUCCIÓN CIENTÍFICA ESPAÑOLA en biomedicina y ciencias de la salud constituye un volumen importante del total de la producción del país. Esta gran área temática constituye algo más del $45 \%$ del total de la producción española ${ }^{1}$. Su importancia y características la hacen peculiar, tal es así, que ha merecido por sí misma la redacción de numerosos estudios e informes destacables $^{2,3}$.

Entre las características más destacables encontramos la de la naturaleza de las instituciones productoras. Mientras que en la mayor parte de los campos temáticos las universidades y el CSIC son los principales productores, la medicina cuenta además con el importante sector sanitario, cuya aportación resulta determinante. Sin embargo, el análisis del comportamiento investigador de estas instituciones se torna difícil, ya que muchas veces no se pueden discriminar con facilidad.

En el "Atlas de la ciencia española" hemos realizado esta tarea, por lo que estamos en condiciones de presentar una propuesta de ranking de instituciones exclusivas del sector sanitario español, para el periodo 1995-2003. La información proviene del ISI Web of Science de Thomson Scientific.

En la tabla podemos apreciar las 50 instituciones más productivas de este sector. Acompaña a cada una de ellas, una serie de columnas de datos que detallamos a continuación. En la primera se presenta la producción total en volumen de trabajos, en la segunda el porcentaje que estos representan del total, en la tercera el porcentaje acumulado, la cuarta la tasa de variación de la producción en los últimos años, la quinta el impacto medio de la producción primaria, la sexta la tasa de variación del impacto normalizado, la séptima el potencial investigador acumulado, y por último en la octava el número de categorías $I S I$ en los que la institución tiene producción.

El primer elemento a destacar es la fuerte concentración de producción en los centros ubicados en los primeros lugares del ranking. Si se toman los diez primeros obtenemos casi la tercera parte del volumen del sector, todos de Cataluña y Madrid. Mientras que para acumular casi la mitad de esa producción sólo se necesitan 21 instituciones.

Las tasas de variación del volumen de producción son, en líneas generales, positivas, lo que indica un aumento del volumen total de producción. Dentro de las más productivas, podemos destacar el caso de la Clínica Universitaria de Navarra (Pamplona), con una Tasa de Variación durante el Período Temporal Estudiado (TVP) de 234,18. Si nos extendemos a toda la tabla, la TVP más alta la presenta el Hospital Universitario Príncipe de Asturias (Madrid) con 822,22 pero, claro está, con un volumen de producción mucho menor. Dentro de las poquísimas instituciones con TVP negativa, destaca la Corporación Hospitalaria Parc Taulí (Sabadell) con -25.
El impacto medio tipificado nos da una idea de la calidad de las revistas en las que han publicado las instituciones. Se observa que, en general, las variaciones son pequeñas y que la mayoría ronda el valor 1 , distribuyéndose de manera uniforme a, lo largo de toda la tabla, aunque destaca el 1,2 del Instituto de Investigación Biomédica Augusto Pi i Sunyer (Barcelona).

En cuanto a la tasa de variación del valor del impacto medio normalizado (TVI), encontramos tanto valores positivos como negativos, repartidos uniformemente a lo largo de toda la tabla. Las TVI van desde los 36,9 del Hospital Universitario Virgen Macarena (Sevilla), hasta los -24,62 de la Clínica Universitaria Hospital Valladolid (Valladolid).

El potencial investigador es la producción de cada institución afectada por el impacto medio tipificado. De esta forma encontramos que los valores son ligeramente mayores a la producción para las instituciones con impacto medio mayor que $1, \mathrm{y}$ menor en el caso contrario. De esta forma el Instituto de Investigación Biomédica Augusto Pi i Sunyer (Barcelona) tiene una producción de 2354 documentos pero un potencial de 2824,64. Pocos son los centros cuyo potencial es menor a la producción.

El número de categorías ISI en las que cada institución presenta producción nos da una idea de la especificidad y diversificación temática de la misma. Los valores parecen repartirse uniformemente a lo 


\begin{tabular}{|c|c|c|c|c|c|c|c|c|c|}
\hline $\mathrm{N}^{0}$ & Institución & Producción & $\%$ & Acum & TVP & IM & TVI & Potencial & $\mathrm{N}^{\circ}$ categ \\
\hline 1 & Hosp Clín \& Provincial (Barcelona) & 6688 & 7,19 & 7,19 & 67,06 & 1,187 & 4,87 & $7.931,59$ & 96 \\
\hline 2 & Hosp Gen Univ Vall Hebrón (Barcelona) & 3456 & 3,81 & 11 & 55,02 & 1,164 & $-0,55$ & $4.023,07$ & 88 \\
\hline 3 & Hosp Univ Sta Creu \& Sant Pau (Barcelona) & 2942 & 3,26 & 14,26 & 19,58 & 1,128 & $-3,51$ & $3.317,74$ & 90 \\
\hline 4 & Ciudad Sanitaria Bellvitge (Barcelona) & 2740 & 3,02 & 17,28 & 11,55 & 1,088 & 0,14 & $2.981,18$ & 108 \\
\hline 5 & Hosp La Paz (Madrid) & 2726 & 3 & 20,29 & 24,06 & 1,047 & 23,13 & $2.853,06$ & 82 \\
\hline 6 & Univ Hosp 12 Octubre (Madrid) & 2545 & 2,81 & 23,1 & 71,43 & 1,06 & $-1,06$ & $2.695,42$ & 82 \\
\hline 7 & Hosp Ramón y Cajal (Madrid) & 2405 & 2,58 & 25,68 & 35,45 & 1,089 & $-0,22$ & $2.617,90$ & 84 \\
\hline 8 & Inst Invest Biomèd Augusto Pi i Sunyer (Barcelona) & 2354 & 2,21 & 27,89 & 151,7 & 1,2 & $-1,53$ & $2.824,65$ & 85 \\
\hline 9 & Carlos III HIth Inst (Madrid) & 2050 & 2,19 & 30,08 & 62,58 & 1,149 & $-4,04$ & $2.352,67$ & 107 \\
\hline 10 & Hosp Gen Gregorio Marañón (Madrid) & 1978 & 2,11 & 32,19 & 27,17 & 1,101 & 12,66 & $2.174,65$ & 74 \\
\hline 11 & Fdn Jiménez Díaz (Madrid) & 1913 & 2,08 & 34,28 & 20,23 & 1,154 & $-14,9$ & $2.206,34$ & 70 \\
\hline 12 & Hosp Univ San Carlos (Madrid) & 1890 & 2,06 & 36,34 & 27,91 & 1,067 & $-3,45$ & $2.017,54$ & 63 \\
\hline 13 & Hosp Univ La Fe (Valencia) & 1878 & 1,81 & 38,14 & 7,26 & 1,023 & $-4,39$ & $1.920,93$ & 89 \\
\hline 14 & Hosp Gen Univ Germans Trias i Pujol (Badalona) & 1650 & 1,78 & 39,92 & 39,67 & 1,105 & $-8,42$ & $1.823,10$ & 68 \\
\hline 15 & Hosp Univ La Princesa (Madrid) & 1608 & 1,46 & 41,38 & 65,38 & 1,168 & 6,93 & $1.876,25$ & 62 \\
\hline 16 & Clín Puerta de Hierro (Madrid) & 1325 & 1,46 & 42,84 & 113,7 & 1,056 & $-4,01$ & $1.399,72$ & 73 \\
\hline 17 & Hosp Univ Marqués de Valdecilla (Cantabria) & 1324 & 1,46 & 44,3 & 60,53 & 1,056 & 5,74 & $1.397,93$ & 63 \\
\hline 18 & Clín Univ De Navarra (Pamplona) & 1321 & 1,42 & 45,72 & 234,2 & 1,092 & $-7,4$ & $1.442,88$ & 72 \\
\hline 19 & $\begin{array}{l}\text { Complejo Hosp Univ Santiago de Compostela } \\
\text { (Santiago de Compostela) }\end{array}$ & 1283 & 1,39 & 47,11 & 114,9 & 1,022 & $-5,55$ & $1.309,86$ & 78 \\
\hline 20 & Hosp Univ Virgen del Rocio (Sevilla) & 1272 & 1,34 & 48,45 & 94,62 & 1,009 & 7,36 & $1.283,14$ & 69 \\
\hline 21 & Hosp Clín Univ Valencia (Valencia) & 1214 & 1,33 & 49,78 & 186,4 & 1,065 & $-15,1$ & $1.291,19$ & 67 \\
\hline 22 & Hosp Cent Asturias (Oviedo) & 1206 & 1,23 & 51,01 & 64,38 & 1,016 & $-3,62$ & $1.225,27$ & 66 \\
\hline 23 & Inst Municipal Invest Méd (Barcelona) & 1117 & 1,08 & 52,09 & 33,33 & 1,175 & 0,77 & $1.312,58$ & 94 \\
\hline 24 & Hosp Univ Miguel Servet (Zaragoza) & 1103 & 1,04 & 53,12 & 150 & 0,963 & 4,79 & $1.060,80$ & 88 \\
\hline 25 & Hosp Gen Univ Valencia (Valencia) & 977 & 1,04 & 54,16 & 82,86 & 1,064 & $-0,83$ & $1.037,00$ & 68 \\
\hline 26 & Hosp Univ Nuestra Señora del Mar (Barcelona) & 951 & 0,99 & 55,14 & 76,81 & 1,099 & 7,97 & $1.044,88$ & 73 \\
\hline 27 & Hosp Reg Univ Reina Sofía (Córdoba) & 942 & 0,97 & 56,12 & 170,3 & 1,13 & $-3,04$ & $1.064,68$ & 62 \\
\hline 28 & Complejo Hosp Salamanca (Salamanca) & 866 & 0,95 & 57,06 & 34,83 & 1,061 & $-23,3$ & 917,779 & 60 \\
\hline 29 & Hosp Univ Virgen Arrixaca (Murcia) & 850 & 0,94 & 58,01 & 75,41 & 0,956 & $-9,08$ & 812,618 & 63 \\
\hline 30 & Hosp Cruces (Barakaldo) & 804 & 0,87 & 58,87 & 89,47 & 1,032 & 0,21 & 828,695 & 58 \\
\hline 31 & Hosp Clín Univ Lozano Blesa (Zaragoza) & 804 & 0,86 & 59,73 & 114,6 & 1,028 & 5,36 & 826,677 & 64 \\
\hline 32 & Hosp Univ Virgen Macarena (Sevilla) & 785 & 0,83 & 60,56 & $-12,79$ & 1,075 & 36,9 & 843,043 & 59 \\
\hline 33 & Inst Valenciano Estudios Salud Públ (Valencia) & 751 & 0,81 & 61,37 & 114 & 1,129 & $-3,37$ & 814,029 & 113 \\
\hline 34 & Complejo Hosp Juan Canalejo (A Coruña) & 729 & 0,73 & 62,1 & 170,3 & 1,04 & $-11,6$ & 758,39 & 58 \\
\hline 35 & Inst Recerca Oncològica (Barcelona) & 695 & 0,7 & 62,8 & 123,9 & 1,17 & 7,81 & 812,875 & 53 \\
\hline 36 & Complejo Hospitalario Carlos Haya (Málaga) & 661 & 0,67 & 63,47 & 65,12 & 1,037 & 1,3 & 685,383 & 63 \\
\hline 37 & Complejo Hosp Virgen de las Nieves (Granada) & 644 & 0,66 & 64,13 & 89,47 & 1,013 & 8,51 & 652,227 & 55 \\
\hline 38 & Hosp Gen Univ Alicante (Alacant) & 618 & 0,65 & 64,78 & 2,78 & 0,981 & $-3,95$ & 605,949 & 50 \\
\hline 39 & Corporac Hosp Parc Taulí (Sabadell) & 608 & 0,64 & 65,42 & -25 & 1,107 & $-15,4$ & 673,34 & 47 \\
\hline 40 & Inst Català Oncol (Hospitalet de Llobregat) & 599 & 0,62 & 66,04 & 216,7 & 1,157 & $-7,28$ & 693,208 & 52 \\
\hline 41 & Hops Clín San Cecilio (Granada) & 591 & 0,55 & 66,59 & 100 & 1,026 & $-3,93$ & 605,074 & 63 \\
\hline 42 & Hosp Univ Príncipe de Asturias (Madrid) & 582 & 0,54 & 67,14 & 822,2 & 1,063 & $-14,2$ & 618,885 & 65 \\
\hline 43 & $\begin{array}{l}\text { Complejo Hosp Dr Negrín (Las Palmas de Gran } \\
\text { Canaria) }\end{array}$ & 497 & 0,54 & 67,67 & 82,86 & 1,069 & $-17,3$ & 531,245 & 45 \\
\hline 44 & Complejo Asistencial Son Dureta (Palma de Mallorca) & 486 & 0,52 & 68,2 & $-18,18$ & 1,048 & 5,45 & 509,371 & 52 \\
\hline 45 & Hosp Donostia (Donostia) & 484 & 0,52 & 68,72 & 311,1 & 1,029 & $-0,05$ & 498,179 & 57 \\
\hline 46 & Hosp Sant Joan de Déu (Esplugues de Llobregat) & 481 & 0,48 & 69,2 & 42,11 & 0,977 & $-10,2$ & 470,084 & 63 \\
\hline 47 & Clín Univ Hosp Valladolid (Valladolid) & 442 & 0,48 & 69,68 & 77,78 & 1,066 & $-24,6$ & 471,206 & 49 \\
\hline 48 & Hosp Univ Mútua Terrassa (Barcelona) & 440 & 0,47 & 70,15 & 300 & 1,168 & $-3,04$ & 512,749 & 55 \\
\hline 49 & Hosp Univ Canarias (Tenerife) & 436 & 0,47 & 70,62 & 183,3 & 1,163 & $-9,66$ & 507,235 & 71 \\
\hline 50 & Hosp Univ Dr Josep Trueta (Girona) & 425 & 0,45 & 71,07 & 184 & 1,112 & $-3,37$ & 472,577 & 42 \\
\hline
\end{tabular}

Tabla 1. Instituciones más productivas

largo de la tabla, aunque obviamente por cuestiones de volumen las últimas presenten menos categorías. Como institución más diversificada encontramos el Instituto Valenciano de Estudios de Salud Pública (Valencia) con 113 categorías, mientras que en el otro lado tenemos el Hospital Universitario Dr. Josep Trueta (Girona) con sólo 42.

Finalmente, y a modo de conclusión, es preciso destacar que el sector institucional que forman los centros pertenecientes al sistema sanitario ha aportado al conjunto de la producción científica española un porcentaje próximo al $30 \%$ anual. Lo que significa que su cre- cimiento medio ha sido similar al del conjunto de los sectores. Ello puede valorarse como muy significativo, habida cuenta del aumento productivo que ha experimentado España en los últimos años. En todo caso, el dato más relevante relativo a la evolución de la producción del sistema sanitario español es el del incremento del número de centros que realizan actividad investigadora, doblándose entre 1995 y 2003. Esto supone un gran desarrollo de la estructura institucional de la investigación en el sector, lo que debe estar teniendo efectos sobre la mejora de los indicadores de producción y visibilidad.

\section{Notas}

1. Moya-Anegón, F. de (dir.); Chinchilla Rodríguez, Z. (coord.). Indicadores bibliométricos de la actividad científica española - 2004. Madrid: Fundación Española para la Ciencia y la Tecnología (FECyT), 2005.

2. Camí, J.; Suñén-Piñol, E.; Méndez-Vásquez, R. I. "Mapa bibliométrico de España 1994-2002: Biomedicina y ciencias de la salud". En: Medicina clínica, 2005, n. 124, pp. 93-101.

3. Camí, J.; Suñén-Piñol, E.; Méndez-Vásquez, R. I. Informe del Instituto de Salud Carlos III-Fondo de Investigación Sanitaria. Disponible en: http://www.isciii.es/mapabiomedico

Informe enviado a EPI por el Grupo SCImago (Imago scientiae o visualización de la ciencia).

scimago@ugr.es

http://www.atlasofscience.net 